\title{
DICOM DATA PROCESSING OPTIMIZATION IN MEDICAL INFORMATION SYSTEMS
}

\author{
ALEXANDR GOLUBEV; PETER BOGATENCOV, AND GRIGORE SECRIERU
}

\begin{abstract}
The problem of storage and visualization of medical images collected by various medical equipment is actual for latest 10 years for every medical institution. On the other hand, access to the medical investigation datasets and solving the problem of personal patient data security is important for scientific community and institutions that require this data. "DICOM Network" project was developed for solving these problems for different actors in the system based on the various customized roles. This article describes the problems and possible solutions for optimization of medical images storing, providing stable and secure access, based on the distributed warehouse for huge volumes of data with different levels of access.
\end{abstract}

Key words: DICOM; Distributed storage system; HPC; Processing algorithms; Radiology; e-Health;

AMS subject classifications. 68M14

1. Introduction. Twenty years ago, after the patient's visit, the doctor had only data on several indicators: general information about the disease, weight, pressure and symptoms. Today a large amount of digital information coming from different sources - from x-ray images to telemetry from implantable devices, such as cardiac monitors. As medical institutions abandon the paper records of the disease, these data are collected more electronically. The availability of large datasets of digital medical information allows doctors to improve diagnoses and health care in general, there is even a new concept - research "in silicon." Information technologies (IT) are now used at all stages of health care, from basic research to the provision of health services, and includes many specializations, such as bioinformatics, clinical informatics and biomedical informatics

Clinical informatics is devoted to the use of IT to improve health care and covers such processes as the preparation of medical bills, the planning of patient care, the allocation of resources for patient care. Clinical decision support systems can, for example, alert the attending physician about the potential interactions of medications, based on the patient's medical history and known allergic reactions.

Modern medical information systems integrate various types of medical equipment. This article describes the actual problems and solutions for optimizing processing and storage of medical radiography investigations. The standard for working with medical images is the DICOM format, which allows storing studies in good quality with the patient's personal data included. The main problem in storing data in DICOM format [1] is caused by the fact that one study can produce more than one gigabyte of data and consists of thousands of images.

DICOM (Digital Imaging and Communications in Medicine) - the standard for processing, storing, printing and transmitting information in medical imaging systems. It includes a description of the file format and network protocol. The network protocol uses TCP / IP in its core for communication between systems. Also, systems that support reading and writing DICOM files can exchange files in DICOM format among themselves. The owner of the standard is the American Organization National Electrical Manufacturers Association (NEMA). It is developed by the Committee of the DICOM standard, consisting of several working groups (WG).

DICOM allows the integration of scanners, servers, workstations, printers, and networking equipment from many different manufacturers into a single PACS (picture archiving and communication system). Different devices are delivered with a document called the DICOM conformance statement, which describes how and what functions the supplied device performs.

The "DICOM Network" [2] project was launched in Moldova in 2015 having a goal is to provide access to investigations for medical staff with the appropriate access rights and as well as patients themselves to the personal radiography investigations. The current realization of the project allowed connecting eleven types of medical equipment to the deployed system. Today the system collects and processes more than 1000 gigabytes of data per month.

${ }^{*}$ Research and Educational Networking Association of Moldova Str. Academiei 5, office 324, MD-2028, Chisinau, Republic of Moldova (galex@renam.md). 
One of the problems that should be solved in DICOM Network project is the problem of storing medical investigations archive on national level that can be considered as Big Data issue. Solution for this issue should take into account the different data access levels. On the one hand, a medical investigation contains personal patient data, which means that data access should be restricted and secured. This could be reached by permission-based categories of users and individual investigations access on supervised approval. On the other hand, data should be accessible by any authorized user, like patient or doctor from any location. One of the main priority is system performance that should allow high speed access to the huge amount of data. Thus, the process of storing and transferring large volumes of medical data can be divided into several components: archiving and storage, retrieval and accessibility of data, data transfer to the end user's workstation and processing the data on the client application.

When medical investigation is completed and DICOM image set imported from the equipment raises the problem of data archiving and data storing. If X-ray photography usually does not contain more than 2-3 images, a tomographic survey can contain up to 1000 slices and take up to several gigabytes on the physical storage. It is easy to calculate that for a large hospital or a large diagnostic center with a daily flow of 500 or more patients, the data volumes will be terabytes per month, but the archive of investigations should be saved for a minimum of three years by law (and even for longer period for practical use). As a result, medical institutions could not archive such a volume of data, because in many cases there are no sufficient capacity available for their storage at the level of one institution.

As far as "DICOM Network" was selected as pilot application for integration into distributed regional VISEEM platform [3], the DICOM Network system architecture was adjusted to the project needs that make possible to increase the number of users and offer access to the DICOM Network for research community. Thanks to VI-SEEM project and gaining access to RENAM[7] computing infrastructure the storage capabilities and processing power is increased in many times and now DICOM Network collecting about 300 investigations per day and over $1 \mathrm{~TB}$ of data per month. All this is possible by using of a number of the developed components: DICOM Server, DICOM Portal, DICOM Viewer, DICOM Audit and others that are enough flexible to cover the requirements of any medical institution or specific scientific community.

2. DICOM File Format Overview. The standard using in "DICOM Network" system for working with medical images is the DICOM format, which allows storing images in good quality. The main problem of data storing in DICOM format in integrated medical data processing systems is caused by generation of multi-gigabyte volume of data and necessity to work with thousands of images. The "DICOM Network" project provides access to investigations for medical staff, patients, and medical researchers with the appropriate access rights to radiography investigations. In the article we describe and analyze possible solutions for optimizing data storing and processing workflows.

2.1. DICOM data structure. DICOM files simultaneously contain both images and additional information about associated patient. Information about the patient and the study cannot be separated from the image itself. This reduces the number of possible errors. The JPEG format file is similarly organized, which can also have additional information describing the image in the file. Any DICOM object consists of a set of attributes, such as the name of the patient, its identifier, the date of the study, etc. Also, one special attribute contains image data (pixel data). Thus, there is no separate header for the DICOM file - only a lot of attributes, including image data. Attributes in the standard are called tags, each tag consisting two fields - the group number and the element number. For example, in the tag numbered 0010, 0010 (tag numbers are written in hexadecimal notation), the patient's name is always included. Each tag has a standard name. 0010, 0010 is called 'Patient's Name'. A list of all standardized tags can be found in the 6th section of PS 3.6: Data Dictionary [4].

The 7FFE, 0010 'Pixel Data' tag can contain one or more images. In the case where the Pixel Data contains more than one image, it is said that the file contains a multi-frame image. In the case of a multi-frame image, one file will contain three or four (for example, several scanned sequences in several places, but at different times) dimensional image. Digital X-ray machines, digital cassette readers give information in the form of a single-frame image. Apparatus like ultrasound, angiographs often give multi-frame images. Old X-ray and magnetic resonance tomography could give single-frame images. Modern tomography (after standardization of the expanded formats DICOM-CT Enhanced and MR Enhanced) can give both single-frame and multi-frame images. 
Image data can be color and monochrome. Color can be in different color coding - RGB, YBR, Palette Color (color palette). Monochrome can be of different depths of gradation of gray (1 - 16 bits). Image data can be packed. The following packing algorithms are standardized: RLE, JPEG, JPEG Lossless, JPEG LS and JPEG 2000. For the whole file it can be applied archiving using LZW (zip) algorithm, however, the realization of such packaging in programs and equipment is rare.

DICOM uses three different schemes for encoding the tags (transfer syntax). The encoding of the file is marked with the corresponding tag in the same file. Schemes are obtained from combinations of two parameters - data representation and byte order coding.

The data representation can be Explicit and Implicit. You need to know how to interpret the data contained in the tag, because this is a simple sequence of bytes. And what kind of data is there - a string, a number, or a sequence of tags (SQ-sequence) is not known in advance. For certainty, the content of each tag has been standardized. Each tag has a standardized representation of tag data (VR, Value Representation) - OB, OW, OF, SQ, UT, UN, etc. When the data is explicitly represented in tags, the VR of the tag is explicitly written. With an implicit view, VR is not written, but is taken from the program table that works with this image.

The order of bytes can be from the oldest to the youngest (big-endian), the record begins with the older one and ends with the youngest, and from the youngest to the oldest (little-endian, "pointed"). The DICOM uses three of the four possible combinations of parameters: Implicit little endian, Explicit little endian, and Explicit big endian. Each tag consists of: group number ( 2 bytes), element number ( 2 bytes), VR ( 2 bytes, in explicit data representation, not implicitly used), tag length (2 or 4 bytes, depending on VR). Some standard VR tags: DA - Date, date; DS - Decimal String, a string representing a decimal fraction; FL-Floating Point Single (4 bytes), a floating-point number of ordinary accuracy; IS - Integer String, integer string; UL - Unsigned Long, unsigned double word, etc. Full information on VR and the principles of encoding tags can be found in the standard - PS 3.5: Data Structure and Encoding [4]. In addition to presenting data (to Value Representation), there is the concept of a plurality of values (VM, Value Multiplicity). VM is not marked in real files, it's just an indication of how much data it is supposed to contain a specific tag. For data represented by strings, the elements are separated by a backslash character ('). Numeric data simply goes sequentially byte - for example, if $\mathrm{VM}=2$, the tag with $\mathrm{VR}=\mathrm{FL}$ will consist of 8 bytes - these are two numbers of usual accuracy. In tables with lists of tags, the VM of each tag is specified. For example, a tag containing one coordinate of a two-dimensional point will have $\mathrm{VM}=2$. The point containing $\mathrm{n}$ coordinates will have $\mathrm{VM}=2 * \mathrm{n}, \mathrm{n} \dot{\mathrm{i}} 0$ (in the standard $2-2 \mathrm{n}$ is written).

2.2. DICOM Protocol and services OVERVIEW. DICOM consists of many different services, most of which involve the exchange of data over the network. The file exchange was added to the standard later and is only a small part of the standard.

DICOM service 'Store' is intended for transferring images or other objects (for example, structured reports - structured reports) between two DICOM devices.

Storage Commitment - This service is used to confirm that relocated objects are successfully stored in the information store. With this message, the person who receives the data - PACS, or the station informs the transmitting device - or the station that the data has been successfully saved and can be deleted.

Query / Retrieve-a service for searching and delivering whole studies or individual objects on a remote DICOM device. You can search for specific filters (for example - research date, patient's name, etc.) of the research or object of interest (most often objects in DICOM are images, but not only) and request its transfer to the local computer.

Modality Worklist allows the device (often devices in DICOM are called modalities, however the same device, for example, a lithotripter, can have several modalities - US, DX) to obtain a list of planned studies. The data on the planned studies contain information about the patients, this allows you to reduce the re-entry of the same information and the accompanying errors.

Modality Performed Procedure Step additional to the Modality Worklist service, which allows the modality to send a report on the success of the research, about the images received, the time of the beginning and the end of the study, the dose received by the patient, etc. Service allows you to get the hospital management more accurate data on the use of the resources of the device. The service, also called MPPS, allows improving the interaction of the modality and the PACS system by providing the system with a list of objects that will be 
sent before the parcel itself.

Printing service allows to send images to print to the DICOM printer, to get a hard copy of the images, most often on tapes. There is a method of standard calibration of printers and monitors, which helps to obtain identical images on different monitors and on a hard copy of images.

2.3. DICOM Files and services OVERVIEW. In addition to directly patient data and research, data points of the image, the files must necessarily contain the so-called meta-information (File Meta Information, tag group 0002). The meta-information indicates how to interpret correctly the contents of the file.

DICOM limits the length of file names to 8 characters, file extensions are not allowed. The names of the files must be such that no information can be obtained from them. These are historically established requirements for maintaining backward compatibility with older systems. On the medium, except files, the dicomdir file should be placed in the root directory. Dicomdir provides general indexed information about all the DICOM files on the media. Dicomdir provides more information about each file than it is possible to fit into the file name.

DICOM files that are not on the media, usually have a .Dcm extension, the media should contain files without an extension.

Some common modalities:

CT - Modality of type Computed Tomography

DX - Modality of type Digital Radiography

MR - Modality of type Magnetic Resonance,

OT - Modality of type Other

US - Modality of type Ultra Sound

XA - Modality of type X-Ray Angiography

3. "DICOM Network" Database Architecture. DICOM Network project based on a number of selfinstalled components that are build using a number of modules. The assemble of components and modules provides a flexible architecture of the DICOM "Network" that is consolidated by DICOM DATA Interface into one radiology investigations database. The system includes Data Storage and Data Processing components distributed between different processing units and storages, which could be customized using specific interfaces. The general architecture of the "DICOM Network" system presented in Fig. 3.1.

Project started from one node located in the Institute of Urgent Medicine (IMU) www.urgenta.md located in Chisinau, Moldova that served only one Institution and had storage element only of 2TB. Then thanks to VI-SEEM project and availability of RENAM computing infrastructure the storage capabilities and processing power increased in many times and now we are collecting about 300 investigations per day and over 1TB of data per month.

- Data from equipment are collecting by the modules integrated in "DICOM Server" that can be installed in the same location with the used medical equipment or can be distributed through different institutions, or even cities or countries.

- All investigations (DICOM Images) are archiving at DICOM Servers, but information about investigation is stored in DICOM Portal (like www.dicom.md) database. Usually various DICOM Servers connected to one DICOM Portal.

- DICOM Portal stores all data like users description, access info, system settings, DICOM Server settings and some others, but not DICOM images it salves. Each institution can deploy DICOM Portal internally using one or several own DICOM Servers.

- DICOM DATA Interface collects information about users and investigations from all DICOM Portals and provides functionality for data exchange and unification.

The functionality of the implemented system covers all necessary workflows for processing and documentation of medical investigations - from images collecting directly from equipment to archiving investigations in the patient medical record [5]. DICOM Network offers extended functionality for enhancing quality of medical management and secured access to investigations. This helps doctors, specialists and penitents to gain access to structured database of medical images, allows documenting images that are collecting from various medical apparatus. At institutional level, the system helps to reduce costs of investigation, raise the quality of provided services. 


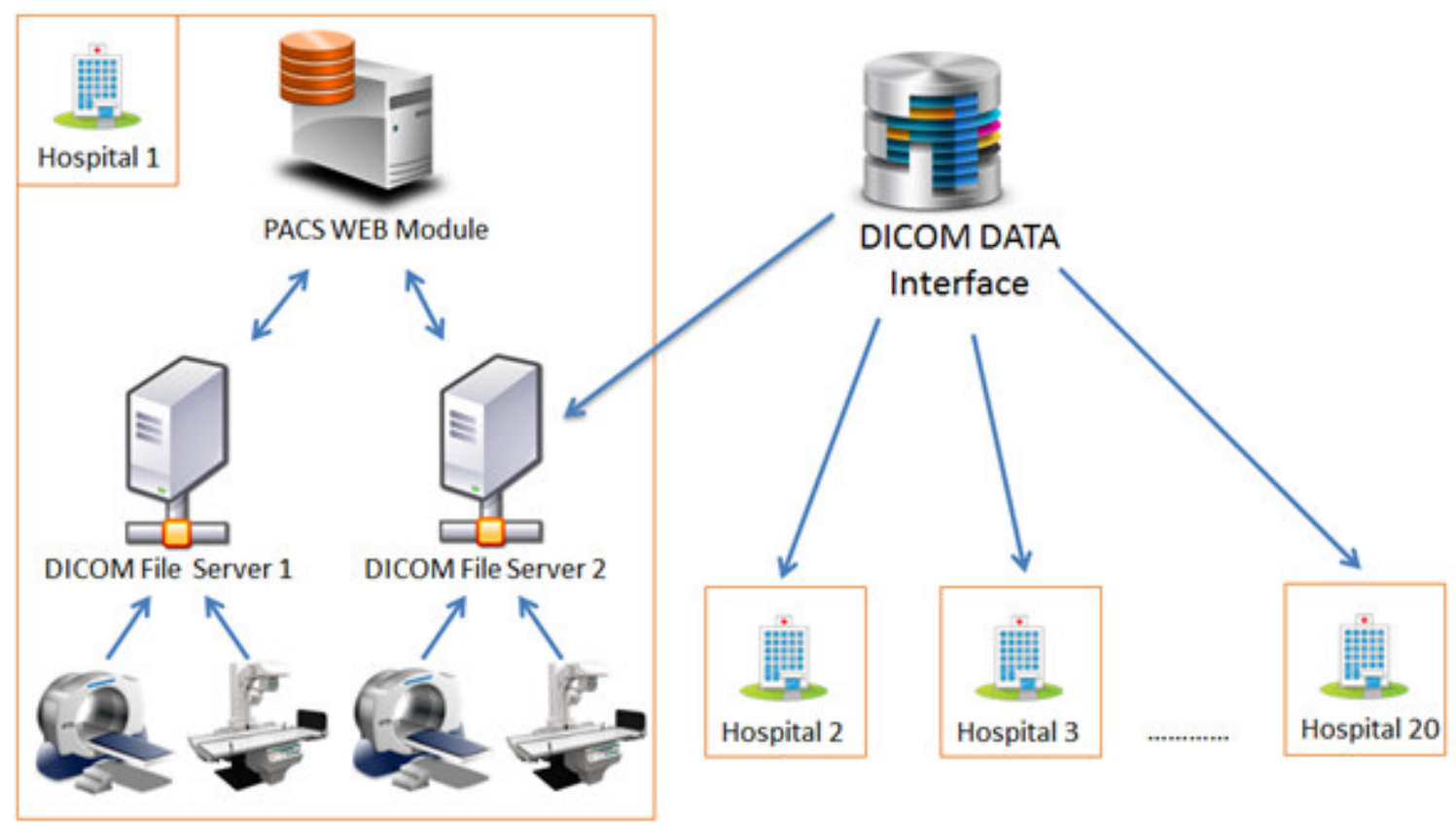

FIG. 3.1. General scheme of DICOM Network architecture

The considering DICOM Network system is already in production operation and can be accessed by link http://www.dicom.md/. The Graphical User Interface (GUI) is presented in the Fig. 3.2.

The system initially was deployed at the National Centre of Ambulance of Moldova (the Institute of Emergency Medicine) and during the first year of functioning has shown it effectiveness and attractiveness for medical personnel that is resulting in:

- Three DICOM Portals operational

- Four DICOM Servers installed

- Eleven types of medical equipment were connected to the "DICOM Network".

- About 300 investigations per day are collecting by the system.

- Over 700 doctors have access to their patients investigations from their working place.

- Over 170000 investigations were searched and downloaded by doctors and radiology specialists during 36 months period.

- Additional budget savings ensured for hospitals due to refusing of printing investigations using expensive consumables.

"DICOM Network" system is actively developing, as far as doctors are interested in operative accessing to radiographic image sets directly from their workplace and institutions want to save money and get technological support for operative and precise decisions. A new types of equipment connected to the system that increases the number of imported investigations. These developments expand the main problem of the systems working with medical images - extreme increasing of data amount that must be preserved.

As far as database is storing personal patient data, security is one of the main key features of the "DICOM Network". We implemented Permission/Role/User system that allows a flexible access to the data. No one investigation or patient can be unauthorized. Each users activity starting with basic access to patient data is logged in the system in separate databased and could not be removed. Each image download is also logged and IP address of the data requester is saved. All of this provides possibility to track and audit each investigation. Access to audit is provided not only for system administrators, but also to responsible persons that have privileged users roles inside the institutions.

The main levels of access are based on following principles: 


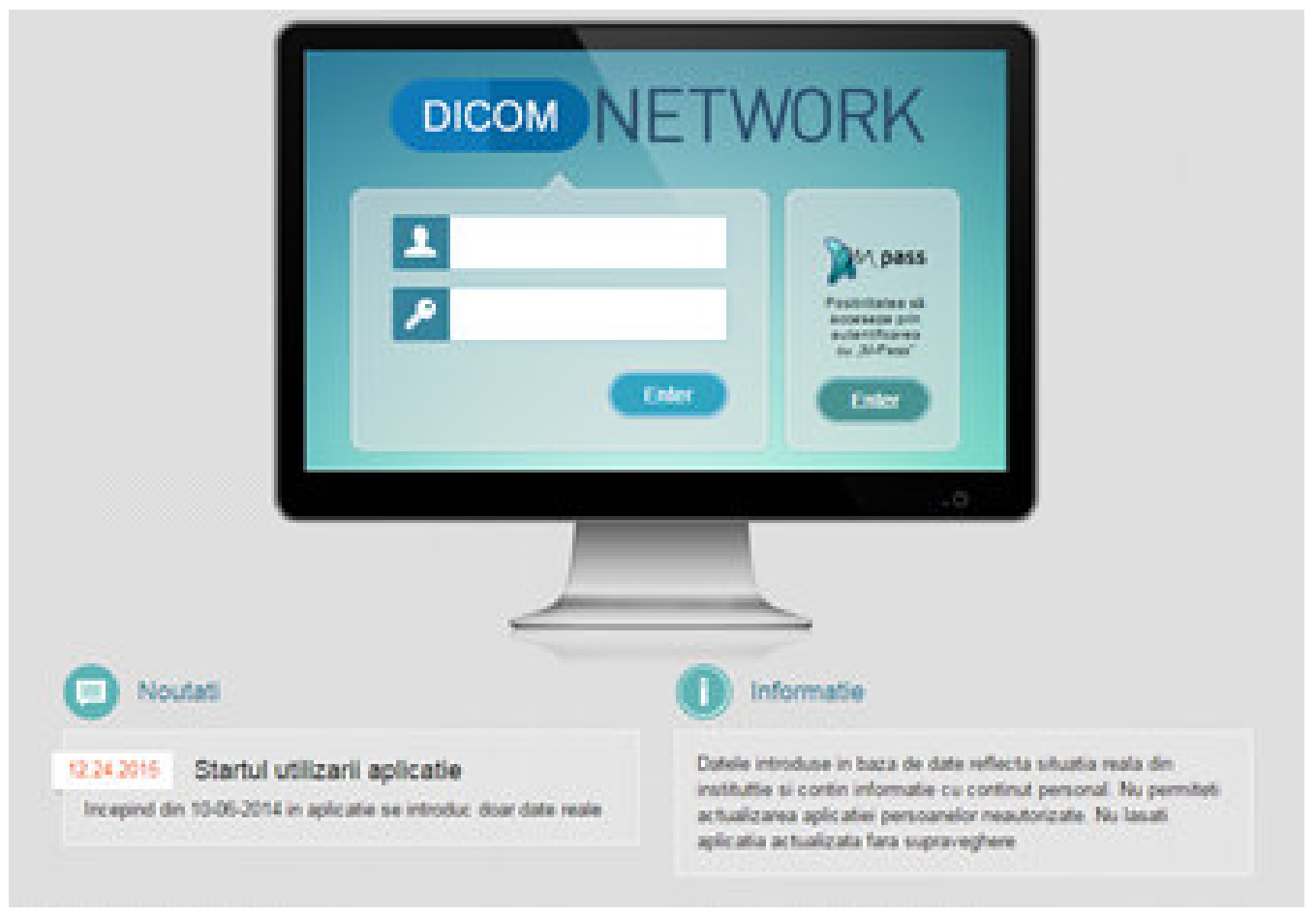

FIG. 3.2. DICOM Network GUI

- Patient can access his investigations based on national ID.

- Doctors can access all the patients investigations in case these are patients that are treated by this doctor.

- Department or Chief of section have access for all patients from his section.

- Specialists from radiology department have access to all data from their institution.

- Scientist and researches have access to the investigations shared by institution, but only in anonymized mode.

- Lawyers and courts can have temporary access to the investigations based on the request of the patient and institution.

- Patient can grant registered access to investigation to another doctor.

Today the data is stored on different DICOM servers and one Storage element. DICOM servers store an archive for the last month and on a common storage is storing a common archive for the last 2 years. This architecture allows providing the fastest access to more recent data that is used more often, while access and downloading images from Storage archive take longer time.

"DICOM Network" is only one information system that works with medical images data storing from different sources on national and international level in this region. Application is based on cloud technologies that makes possible to distribute it on the different locations and dynamically increase resources on demand. One of the benefits of this system is that it is using resources of scientific cloud infrastructure that makes possible to access the investigations both by specialists for daily-based activities and by scientists for research. The other applications that are working in this domain are restricted by their using in one specific institution and do not provide tools for secure data exchange inside the application or organizations. Most of the Information systems based on the open source software and usually these products have insufficient quality or limited functionality, or specialized PACS provided by the equipment supplier that is adopted only to the target equipment without ability of extension in large informational systems.

One of the similar to DICOM Network system is "NeoLogica" software solution. "NeoLogica" designs and develops advanced medical imaging software solutions since 2002. The benefit of "NeoLogica" software is 


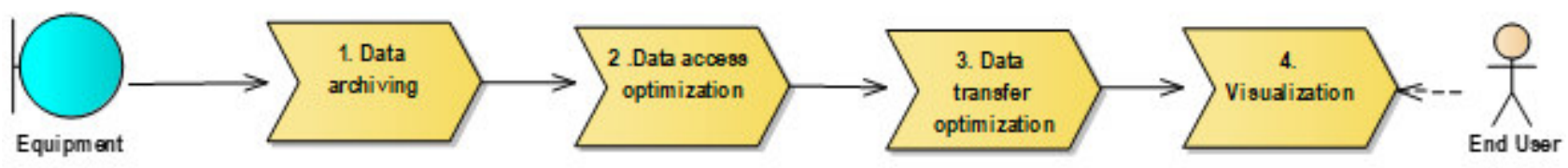

FIG. 4.1. DICOM Network distribution using VI-SEEM platform

also cloud-based architecture that is using cloud hosting solution in Amazon cloud. However, national laws of personal data protection in the most countries, organizations and hospitals is not accepting storing the sensitive data abroad, on Amazon servers, also it increasing costs of data transfers.

4. Main System Optimisation Objectives. First, to describe the solution for DICOM data optimization, here we overview of the algorithms and processes that are necessary for any information system working with radiography images. To obtain the source images, DICOM provides functionality for the import of data directly from the equipment. After importing the image, you must process and save it on commonly accessible storage. As a result, a medical research database available to the authorized user is created. The user accessing the database should receive data through the local or wide area network, and then using the visualization application to work with DICOM images on his workplace.

Thus, the problem of data handle optimization for this kind of information systems can be divided into three stages. First, when you import and write the source files on storage, you need to archive the data to minimize it volume saving the quality of the images. Secondly, when accessing data, it is necessary to transfer the data to user as quickly as possible while optimizing the data format to reduce the amount of transmitted images. Thirdly, the data should be optimized to speed up its loading and processing on the local processing facility.

On the diagram below Fig.4.1 are showed full process of images optimization that is representing by four sequence steps - Archiving, Access, Transfer and visualization:

In the subchapters below are described assembly of solutions for each image lifecycle step optimizations. The proposed algorithm saves $100 \%$ original image quantity, optimize the storage elements and reduce the transferred size of the thumbnails, that could be zoomed on demand. On the final step of visualization server prepares the image set in such way that it could be visualized using low performance processor such as PC or mobile device.

4.1. Data archiving. As it was mentioned above, one radiographic examination can vary from 1 to more than 1000 images. Thus, the investigation can consist (has a size) of more than one image. Of course, the most interesting and useful is archiving of large tomographic studies with a large number of slices. First, let us look at the structure of the DICOM file. In general, each file contains lines of the patient's metadata and the image itself in the raw format. Since during DICOM file processing all meta-information is written in the database, then when archiving we can discard metadata and save only the image. If necessary, it will be possible to restore this data and generate a DICOM file on client request, or use the new file format when transferring it to specialized application like DICOM Viewer. Of course metadata is only a small part of the file and takes only a couple of kilobytes, but it should be taken into consideration, that metadata for one investigation is almost identical, so for a set of data in 1000 slices it will be possible to save significant amounts of physical storage space. On the other hand, excluding the patient's personal data from the original files significantly reduces the possibility of personal data leakage. This approach also allows to create and transfer of impersonated data.

Archiving of image itself is already a much more complicated task that requires complex data compression algorithms. It should be taken into account that it is necessary to exclude loss of image quality since each pixel is important for data processing and visualization. Based on analysis produces we found that archiving an individual slice without loss of image quality does not give a tangible result, but it is worth noting that the proportion of individual slices is small enough and refers mainly to x-rays, when one investigation consists of 1-2 images. The quantity of this data type that is stored does not exceed 1\%. Analysis of more complex tomographic surveys with 100 or more slices shows that standard algorithms of video image compression could 

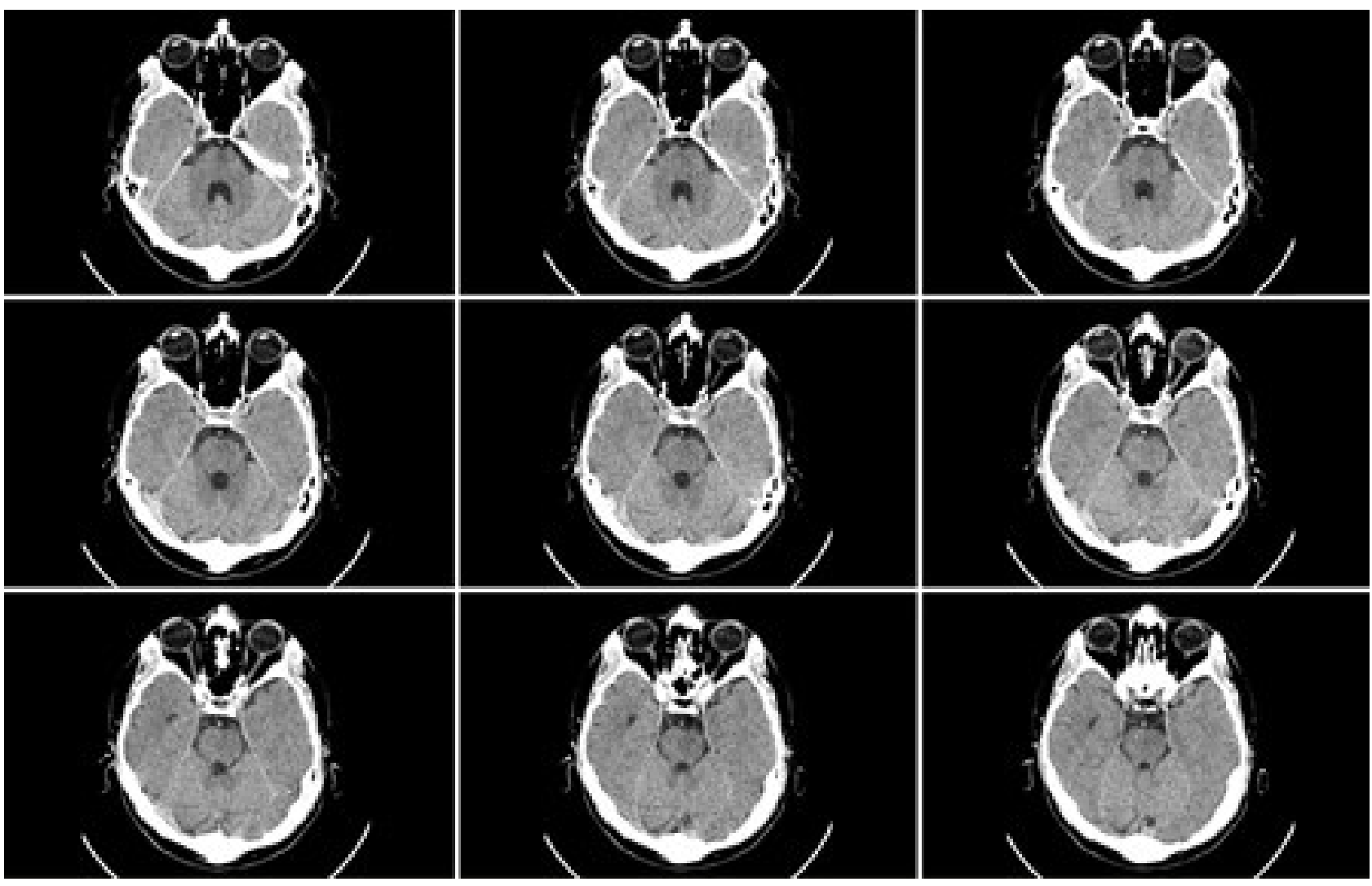

FIG. 4.2. Similar radiology investigations slices

be applied for their archiving, since the changes between slices (frames) are insignificant. In Fig. 4.2 presented the "neighboring" nine slices, the differences in adjacent slices are so small that you can confidently convert these slices into video stream where only the differences for pictures are retained and not the raw format for each image. This way, you can reduce the amount of images with multiple slices by 10 or more times. Of course, you need to take into account that for archiving you will need considerable computing capacity, which involves the use of high-performance computing systems such as HPC[8] or computing GRID[10].

4.2. Data access optimization. Another problematic aspect of data storage is the distribution of data among various data storage systems with different levels of security[10] and access speed. As already mentioned above, storing data in one centralized system is not effective for a number of reasons. To solve this problem, we proposed a distributed data storage system showed in Fig. 4.3.

The main components of the proposed distributed storage system architecture are:

- Local Storage Since the equipment connected to front-end DICOM server directly, the server must be located both in the local network and in the wide-area network. The medical equipment should be located only in the local network. For solution of this problem is using a local server, physically located near to the equipment (one per cabinet or laboratory). This server receives the DICOM data and performs the primary data processing. On one hand, this server provides the fastest access to data; on the other hand, a real storage capacity of this server is not exceeds 1-2 terabytes, which implies the stored data availability within 1-2 months. This allows high-speed access to the latest data. After a predefined period, the data archived and transferred to the next institutional storage level ("Laboratory Storage") or to "External Storage" level.

- Laboratory Storage Storage element for one institution, available in the local network of the organization. This storage has extended parameters, but usually they are limited too and can store data for 


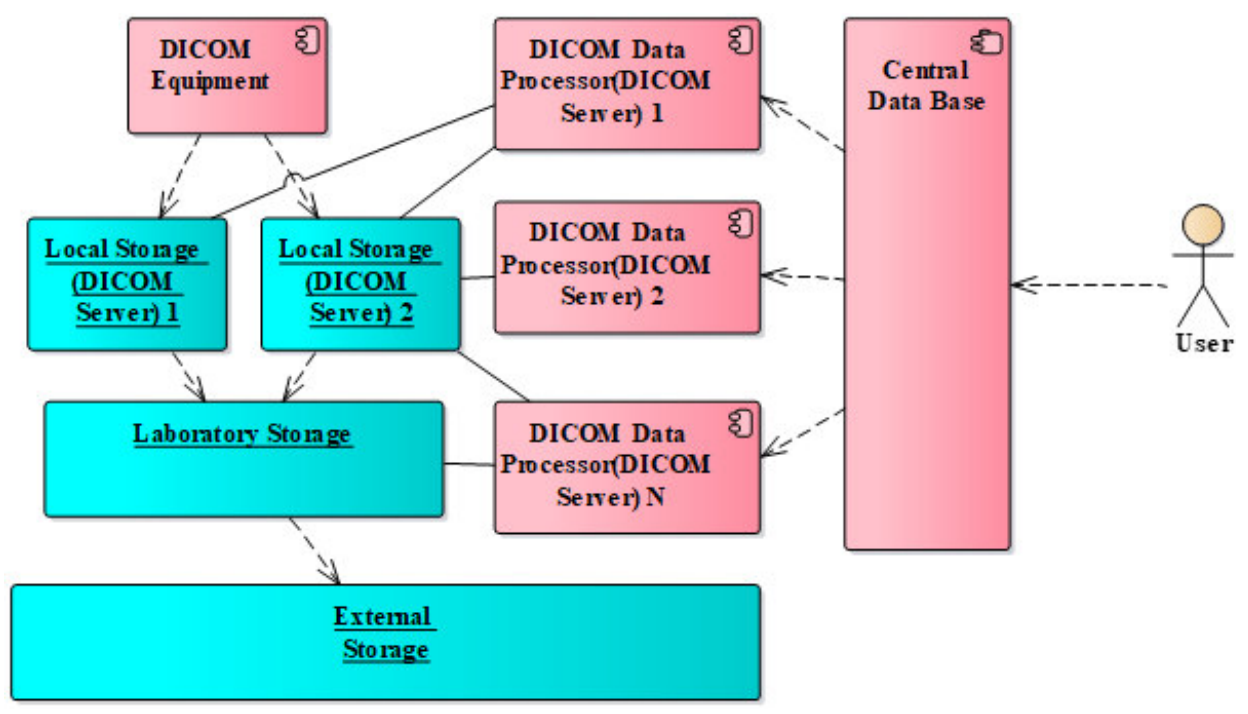

FIG. 4.3. Distributed Big Data Storage

1-2 years.

- External Storage Distributed storage, located partly inside and mainly outside of the organization where the investigations were created and interoperable through the global network infrastructure. This type of storage has the lowest access speed, but it can store a huge distributed archive of data at the national or international levels

4.3. Data transfer optimization.. Regardless of the storage location for their visualization, images must be uploaded to the end user's computer. At the same time, regardless of the transmission channel, which can be a high-bandwidth local network or the global Internet network with unpredictable QoS, data transactions can be very large, and the speed of downloading may influence on quality of medical services and on convenience for medical doctor of the information system using.

The main solution to minimize delays is certainly implementation in the system procedures of archiving and decompressing of data described above, but it is worth taking into account the following opportunities for transmission optimization:

- Preparing a data packet taking into account the permission from client application of the receiving specific data sets. That is, if a user views a survey on a low-resolution mobile device, there is no sense in sending full-screen images at the maximum resolution.

- Ability to load a specific slice in the maximum resolution. At the request of the client application, it is necessary to provide the possibility of sending a particular image at the maximum resolution, for example, if necessary to use zoom in / zoom out.

- Loading data in background with a separate thread. In this case, the end user can begin to visualize not a complete set of data, while the full set will be loaded asynchronously and displayed as the load is finished.

- Caching data at the client and server level.

All of the above can significantly speed up the transfer of data and make the application for visualization more user friendly.

4.4. Preprocess of data for visualization.. After uploading data to the end user's computer, the application that implements the DICOM image rendering must process the data for visualization. In this case, all data must be loaded into the operative memory for fast processing. It should be noted that the DICOM Viewer (the application that displays the DICOM images) should not only display the image and change the slices, but also perform more complex operations - from drawing to building 3D models, modeling and 


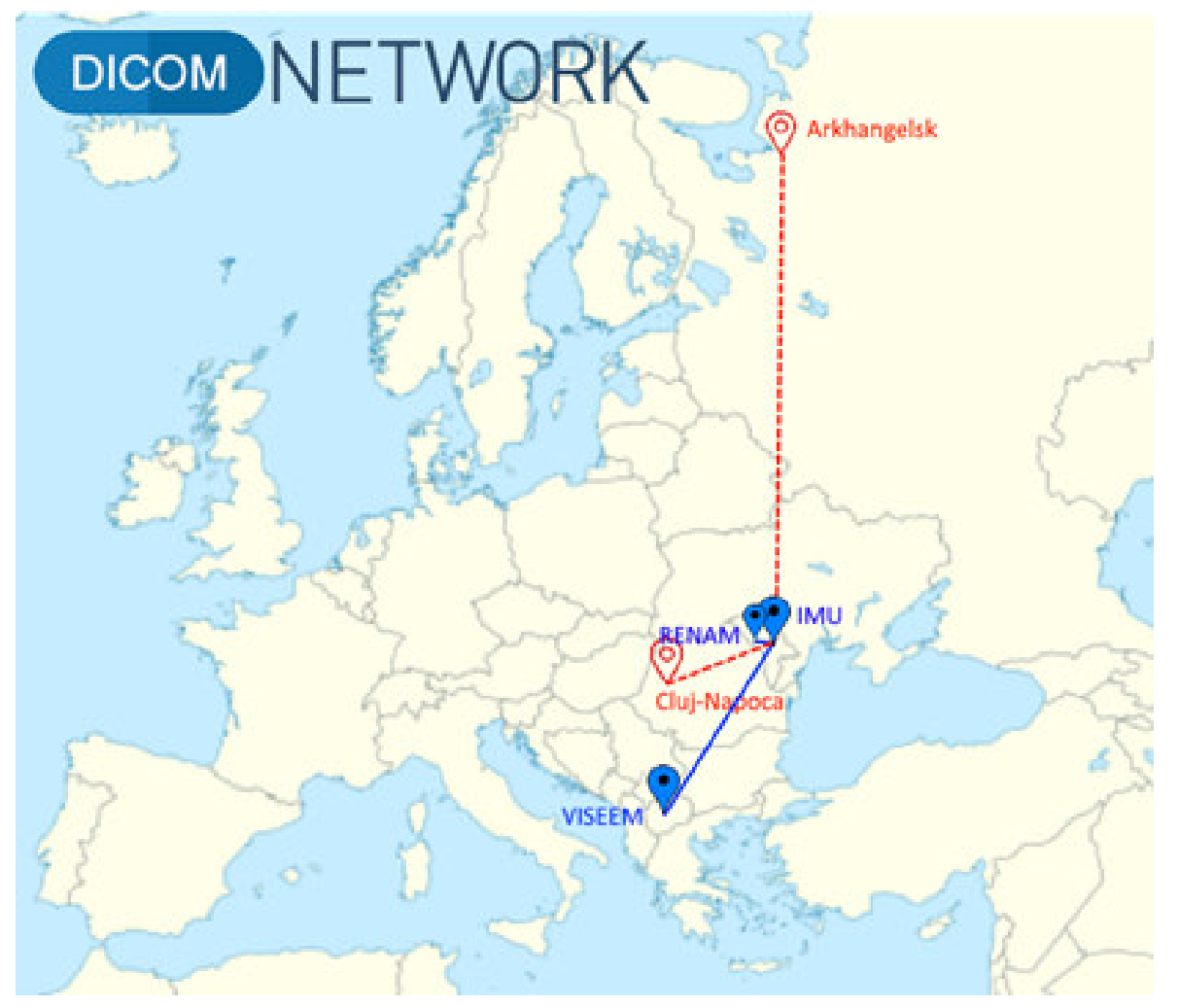

FIG. 5.1. DICOM Network distribution using VI-SEEM platform

representing the tissues. Not every desktop computer, much less of mobile devices have satisfactory computing capabilities. That is why the above-mentioned operations may take a long time.

The solution for this problem should be preparation of data for visualization on the server part of the system. Considering the use of high-performance systems, on the multiprocessor server can be build and transferred ready-made models that will not require complex conversions on the client facility.

5. Integration of "DICOM Netwrk" in VI-SEEM Platform. As far as "DICOM Network" was selected as pilot application for integration into distributed regional VI-SEEM platform [5], the DICOM Network system architecture was adjusted to the project needs that made possible to increase the number of interested users and provide access to the system for wider community for research purposes. The current number of collected investigations in the system is over 187 000, that are regularly requested. Many medical institutions and different medical staff are working with this system. Two medical institutions refused using any other PACS and archiving system and now are using exclusively DICOM Network for patient workflow.

Now installed three DICOM Portals and four DICOM Servers in two countries: Moldova and Macedonia. The clinic from Cluj-Napoca in Romania and Federal University named after M.V. Lomonosov in Russia expressed their interest to install DICOM Network. The current state of "DICOM Network" distribution is show in the Fig. 5.1.

At present available the following DICOM Portals:

- http://dicom.md/ in IMU

- http://renam.dicom.md/ in RENAM

- http://viseem.dicom.md/ in Macedonia (VI-SEEM project).

As was shown above, the solution for storing such large volumes of data is the distributed storage for images archiving. One solution is to participate in international projects and use storage elements from the resources available for the project realization. This cooperation is mutually beneficial for both "DICOM Network" and 


\section{DICOM NETWORK}

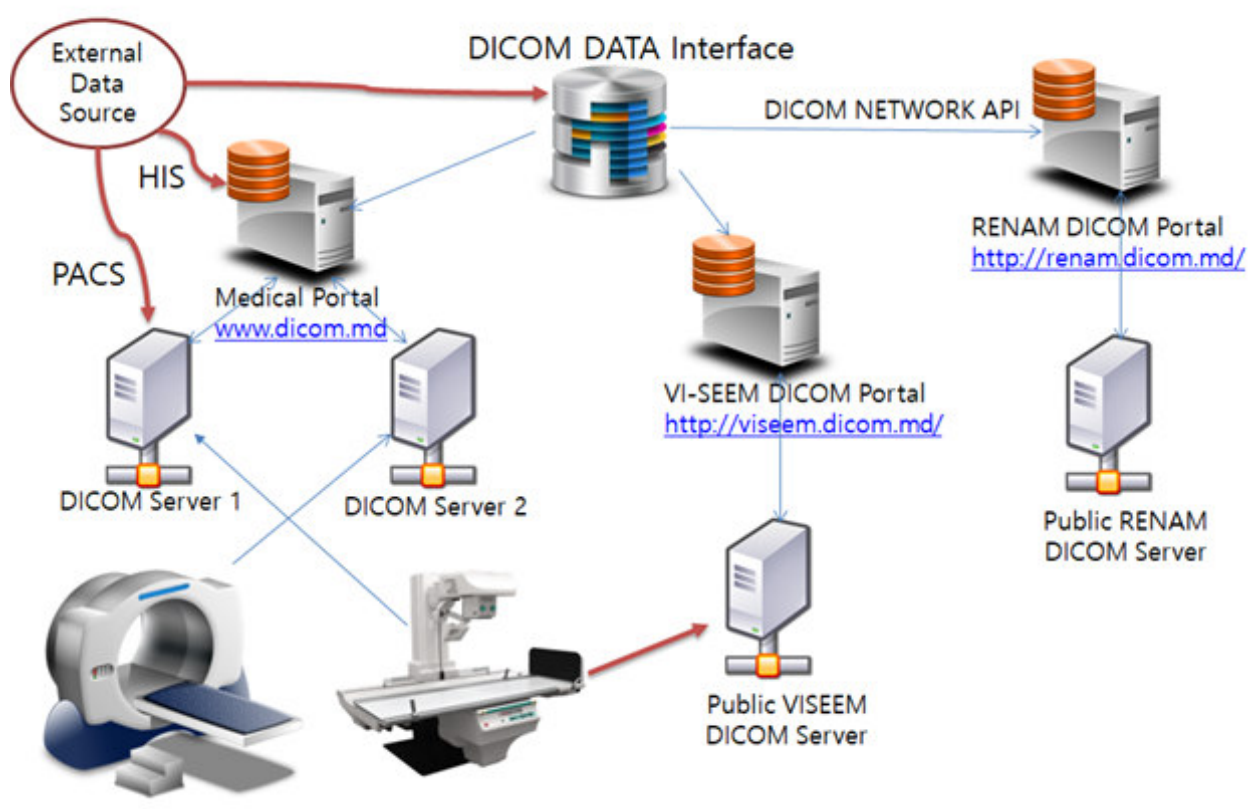

FIG. 5.2. DICOM Network architecture for integration with VI-SEEM platform

the VI-SEEM platform. VI-SEEM provides project resources while "DICOM Network" datasets for research community. Of course, all data collecting on the VI-SEEM servers is impersonated.

In the framework of VI-SEEM project an instances of DICOM Portal and DICOM Server were installed on the Macedonian server and are available on using following link http://viseem.dicom.md/. This server, that is available for research community have been configured for both collecting (DICOM Server installed) and distributing the medical images using DICOM Portal. Each organization can register on this server their equipment and then medical images data will be uploaded and accessible using DICOM Portal. Based on assigned role and permissions data can be anonymized, shared or distributed through other portals according to user defined rules in the system management interface of the portal that is accessible from the web browser. Now 900 gigabytes with anonymized datasets were already uploaded to the server and are available for researchers.

VI-SEEM project deploys and offering user-friendly integrated e-Infrastructure platform for Scientific Communities in Climatology, Life Sciences and Cultural Heritage for the South-Europe and Mediterranean regions by linking compute, data and visualization resources, as well as generalized services, software and tools. The regional infrastructure deployment concept is presented in Fig. 5.2

In the Fig. 5.2 presented the concept of connecting national DICOM Network application, that it is containing existing DICOM Portal http://dicom.md/, with DICOM Portal installed by using VI-SEEM platform resources[8]. DICOM DATA interface grants the interconnectivity for different users of the both portals and allows displaying DICOM investigations using the both portals interfaces. Public DICOM Server grants possibility for any authorized VI-SEEM platform user to add and retrieve the investigations from DICOM Network application and use the developed facilities based on configured and granted access rules.

VI-SEEM platform will offer possibility to install and configure publically available DOCOM Portal that can be used by any interested institutions to store, access and share medical images. Setting up public DICOM Portal instance will increase the level of access to DICOM investigations and will help to make DICOM Network services available to regional medical research and practicing community. 
6. Concusions. Now "DICOM Network", although actively developing, is still far from realization the whole potential incorporated into the system. Taking into account the growing number of medical equipment and the trend towards modernization and computerization of various health facilities, the system will be able to receive and will have to process dozens of terabytes of source information. The storage and subsequent transfer of such large amounts of data is an expensive process, which effective development impossible without optimization and new approached realization. On the other hand, for the successful development of the system it is necessary to accumulate and provide access to the archive not only for 3 years, as requested by law, but for ten or more years to monitor the patient's condition changes and maintain a full medical record. It is also necessary to take into account the need for backup copies of such important information. It is easy to calculate that even for rather small country as Republic of Moldova the volumes of data are too large to store them in an unprocessed form. Thus, the issue of data optimization and using effective archiving algorithms are the key factors for development of e-Health systems.

The benefits of implementation of new effective archiving algorithms are determining the following four directions of medical information systems development:

- Reducing the costs of medical data storage and maintenance;

- Reducing internet traffic for data access and in such way reduce the costs for data transfer;

- Increasing the quality of radiology services for patients.

- Solving the main problem for DICOM images database growth insufficient space for permanently increasing amount of imagistic data.

Radiology medical investigations services are offered by majority of medical organization starting from located in small villages to huge laboratories in the specialized medical centers. In any medical institution effective archiving algorithms should be in a great demand, because on the one hand they allow reduce the costs, save organizational budget [6], and on other hand increase quality of offered services, open new opportunities for collaboration with other institutions and making joint research. Of course, the most perspective consumers of the proposed solution are large diagnostics centers in governmental and private medical organizations that have modern equipment and make huge number of investigations that require archiving and transferring to other medical institutions. However, the implemented solutions will be also interesting for small hospitals that do not have their own equipment but anyway need to have access to the radiology investigations for their patients. Using the proposed solution these small hospitals will obtain possibility to have access to the investigations that were done in external institutions like specialized diagnostics centers.

Datasets collecting in the "DICOM Network" system will provide new opportunities for researchers. Although the system is now in production stage, functionality of the "DICOM Network" is permanently enhancing. During the process of the system implementation beneficiaries have ability to specify their specific necessities for providing additional features and services, such as:

- Studying and realization of new methods for optimization of data transfer and archiving.

- Image preprocessing and detection of anomalies.

- Incorporation of expert systems to help making diagnoses for doctors.

- Development of open APIs for "Dicom Network" to collect, archive, access and jointly process medical images at international level using distributed computing infrastructure.

Algorithms and solutions described in the paper are open for joint development and can be applied to various medical information systems. They do not depend on any specific project, since the developed approach involves tight integration with the open DICOM standard and mostly complements it rather than modifies.

\section{REFERENCES}

[1] A. Golubev, P. Bogatencov, G. Secrieru. Optimal Methods of Storage, Transfer and Processing of DICOM Data in Medical Information Systems, International Conference on Distributed Computer and Communication Networks 2017, $\mathrm{p}$ 269-280

[2] A. Golubev, N. Iliuha, P. Bogatencov DICOM Network services DICOM data exchange solution integrated in the regional VI-SEEM infrastructure, Smart Technologies, IEEE EUROCON 2017-17th International Conference on, 558-563

[3] A. Golubev, P. Bogatencov, G. Secrieru. Updating DiCOM Network Architecture for its Integration at International Level, Networking in Education and Research, 15th RoEduNet IEEE International Conference, Bucharest, Romania, 7-9 September 2016, pp. 161-166. ISSN 2068-1038. 
[4] DICOM Digital Imaging and Communications in Medicine, Published by National Electrical Manufacturers Association. PS 3.6-2011.

[5] P. Bogatencov, N. Iliuha, G. Secrieru, A. Golubev. DiCOM Network for Medical Imagistic Investigations Storage, Access and Processing, "Networking in Education and Research", Proceedings of the 11th RoEduNet IEEE International Conference, Sinaia, Romania, January 17-19, 2013, pp. 38-42. ISSN-L 2068-1038

[6] A. Golubev, P. Bogatencov, G. Secrieru, N. Iliuha. DICOM Network - Solution for Medical Imagistic Investigations Exchange, International Workshop on Intelligent Information Systems. Proceedings IIS. 13-14 September, Chisinau, IMI ASM, 2011, pp. 179-182. ISBN 978-9975-4237-0-0

[7] P. Bogatencov, G. Secrieru, N. Iliuha, N. Degteariov, G. Horos New developments of Distributed Computing Technologies in Moldova, CEUR Workshop Proceedings. Selected Papers of the 7th International Conference Distributed Computing and Grid-technologies in Science and Education. Dubna, Russia, July 4-9, 2016; Vol-1787, urn:nbn:de:00741787-5, pp. 20-25. ISSN 1613-0073

[8] N. Degteariov, Bogatencov P., Iliuha N., Horos G DEPLOYMENT OF THE SCIENTIFIC CLOUD COMPUTING INFRASTRUCTURE IN MOLDOVA, Proceeding of the 5th International Conference "Telecommunications, Electronics and Informatics", May 20 23, 2015, Chisinau, UTM, 2015, pp. 27-29, ISBN 978-9975-45-377-6

[9] A. Golubev, P. Bogatencov, New Trends in Research and Educational Networks Security Teams Operation. An Overview of Automated Tools for CERT, Proceedings of ITSEC-2012 International Conference on Information Technologies and Security, 15-16 October 2012, Chisinau: NCAA, 2013, 181-187. ISBN 978-9975-4172-3-5

[10] N. Iliuha, A. Altuhov, P. Bogatencov, G. Secrieru, A. Golubev, SEE-HP Project Providing Access to the Regional High Performance Computing Infrastructure, Proceedings IIS "International Workshop on Intelligent Information Systems", September 13-14, 2011, Chiinu, 183-186. ISBN 978-9975-4237-0-0.

Edited by: Aneta Karaivanova

Received: Jan 5, 2018

Accepted: Apr 12, 2018 